\title{
End Rule
}

National Cancer Institute

\section{Source}

National Cancer Institute. End Rule. NCI Thesaurus. Code C82565.

An axiom that indicates the end of an event, visit or study element. 